\title{
A real-time, selective, and low-cost strategy for detection of trace level spike-protein from SARS-CoV-2 with application to cold-chain food quarantine
}

Haochen $\mathrm{Qi}^{1} \dagger$, Jian Zhang ${ }^{1,2} \dagger$, Jayne $\mathbf{W u}^{3} \bowtie$, Xin Fang ${ }^{1}$, Wenci Zhao ${ }^{1}$, Fangshuo You ${ }^{1}$, Ying Chen ${ }^{4}$, Lei Zheng ${ }^{2} \bowtie$

1 School of Electronic Science and Applied Physics, Hefei University of Technology, Hefei 230009, China.

2 School of Food and biological Engineering, Hefei University of Technology, Hefei 230009, China.

3 Department of Electrical Engineering and Computer Science, The University of Tennessee, Knoxville, TN 37996, USA.

4 Agro-product Safety Research Centre, Chinese Academy of Inspection and Quarantine, Beijing 100123, China.

† These authors contributed equally: Haochen Qi, Jian Zhang.

凶 e-mail: jaywu@utk.edu, Izheng@hfut.edu.cn

Due to the friendly temperature for virus survival, SARS-CoV-2 is frequently found in cold-chain foods, posing a serious threat to public health. Utilizing an interdigitated microelectrode chip modified with an antibody probe, and integrating dielectrophoresis enrichment with interfacial capacitance sensing, a strategy is presented for detection of trace level spike-protein from SARSCoV-2. It achieves a limit of detection low to $2.29 \times 10^{-6} \mathrm{ng} / \mathrm{mL}$ in $20 \mathrm{~s}$, with a wide linear range of $\left(10^{-5} \sim 10^{-1} \mathrm{ng} / \mathrm{mL}\right)$ and a high selectivity of $6.02 \times 10^{5}: 1$. The cost for single test can be controlled about 1 dollar. This strategy provides a competitive solution for real-time, sensitive, selective and large-scale application in cold-chain food quarantine.

Keywords: SARS-CoV-2, spike-protein, cold-chain food, immunosensor, interfacial capacitance 
The contamination of pathogens in food and environment has always been a serious problem worldwide for food safety, entry-exit quarantine, epidemic prevention and public health management. Although viral epidemics are less reported for food safety compared with bacteria [1], their transmissibility is not weak at all. More typical examples have been showing their destructive power, such as large outbreaks of hepatitis A $[2,3]$ and norovirus infection $[4,5]$. As expected, these viruses are detected in various foods as well as waters $[1,6,7]$, which exacerbates the spread of the disease. Therefore, the viral contamination detection in food poses a more great challenge in recent decade [8].

Since winter 2019, the outbreak of a so-called coronavirus disease 19 (COVID-19) has become a global pandemic, with the culprit being a new SARS coronavirus (SARS-CoV-2) [9]. Although there is not yet a direct evidence to indicate the foodborne characteristic of SARS-CoV-2, food contamination of SARS-CoV-2 may present a risk for virus spreading, which attracts considerable attention in many countries [10-12]. SARS-CoV-2 virions adhering on solid surface are reported to be stable, with a viability up to longer than $72 \mathrm{~h}$ (on plastic) [13]. As a matter of fact, besides the wet markets providing a breeding ground for the virus [14], positive identification of SARS-CoV-2 on cold-chain foods are frequently reported in China recently, including the confirmed SARS-CoV2 virions on plastic packing. Due to the below $0{ }^{\circ} \mathrm{C}$ temperature during the cold-chain transportation, the viruses can have a longer survival time than in room-temperature environment. On the other hand, the virus concentration may be of trace level, while a large number of samples needs to be tested. Therefore, sensitive, fast and low-cost SARS-CoV-2 detection is urgently needed for cold-chain foods.

For virus detection in food, there are mainly two types of techniques, culture/counting methods and polymerase chain reaction (PCR) based methods $[7,8]$. Because the culture process is very time-consuming, PCR based methods have become a mainstream approach $[8,15]$. Numerous works on various virus detection by PCR or reverse transcription (RT)-PCR in food have been reported, including hepatitis A [15-17], hepatitis E [18], norovirus [15-17]. Although PCR techniques have been developed for more than 30 years, the operation is still complicated, and the required turnaround time cannot be shorter than several hours [15]. Based on the good specificity and versatile sensing mechanisms, bio-probe based sensors for virus detection are becoming more practicable in recent years as promising approaches [19-21]. Also due to the high specificity, 
reproducibility, and stability, antibody based detections of causative agent are continually adopted in food safety detection $[29,30]$.

A SARS-CoV-2 virion binds directly with its receptor through spike (S-) protein on its surface, and the subunit of S1 serves as the receptor-binding domain (RBD) [22-24]. As a result, S-protein ( $\mathrm{S} 1$ subunit) is selected as a preferred biomarker for SARS-CoV-2 detection [25-27]. Because the S-protein existing on the periphery of the virion is exposed on the food surface independent of body infection and viral assembly, at a quantity more than viral RNA itself, it can act as a good biomarker for food contamination of SARS-CoV-2. For specific recognition of certain antigen, antibody is considered as a promising probe due to the usually high affinity between antigen-antibody [28].

Here, an approach for trace S-protein (S1 subunit) detection associated with cold-chain food is developed for real-time SARS-CoV-2 contamination screening. An anti-SARS-CoV-2 S-protein antibody is immobilized on a low-cost interdigitated microelectrode (IDME) chip. Then an alternating current (AC) signal is applied to the IDME to induce a dielectrophoresis force on the protein particles. Thus, the S-proteins are rapidly driven toward the IDME surface in the microfluid and captured by the antibodies. Utilizing the electrical double layer capacitance at the IDME surface as an ultra-sensitive indicator, the limit of detection (LOD) reaches $10^{-6} \mathrm{ng} / \mathrm{mL}$, while the time from sample to result is as short as $20 \mathrm{~s}$ due to effective target enrichment embedded into the detection process. Meanwhile, the detection is specific against the interferences, demonstrating a satisfactory selectivity in S-protein recognition. This report constructs a cost-effective immunosensor as well as a simple detection strategy for real-time, selective, and large-scale screening of SARS-CoV-2 for cold-chain food quarantine.

\section{Results}

Dose response from S-protein in PBS buffer. The core device for the presented approach is an IDME immunosensor, and the calibration for this sensor should be performed before its application. The S-protein is tested with tenfold increase from $10^{-5}$ to $10^{-1} \mathrm{ng} / \mathrm{mL}$ in $0.1 \times \mathrm{PBS}$ solution. Here the upper-limit concentration of $10^{-1} \mathrm{ng} / \mathrm{mL}$ is determined by observing the saturation phenomenon [31, 32] at $1 \mathrm{ng} / \mathrm{mL}$ illustrated in Fig. S1a and Fig. S1b in supplementary information.

The typical transient curves of normalized capacitance vs. time acquired from the sensors are 
presented in Fig. 1a The curves can be clearly differentiated between different target concentrations, and exhibit increasingly downward slopes with the increase of S-protein concentration in $0.1 \times \mathrm{PBS}$. This indicates the sensor's primary performance of output monotonicity In Fig. 1a, the linearity of the transient curves become worse after $10 \mathrm{~s}$ when the concentration is high. Therefore, the response time of this sensor is chosen to be $20 \mathrm{~s}$.

The dose response is shown as Fig. 1b, where the response is normalized by the initial capacitance, and $\mathrm{dC} / \mathrm{dt}$ represents the change of normalized capacitance in 1 minute's duration, which is found by least squares linear regression method. The calibration curve, $Y(\%)=-2.66 \lg X$ $(\mathrm{ng} / \mathrm{mL})$, demonstrates an excellent semi-log linear relationship between the target concentration and the response from $10^{-5} \mathrm{ng} / \mathrm{mL}$ until $10^{-1} \mathrm{ng} / \mathrm{mL}$, with a correlation coefficient $\left(\mathrm{R}^{2}\right)$ of 0.994 . With the cut-off line defined as a response value three standard deviations from the background, the LOD is calculated to be $2.29 \times 10^{-6} \mathrm{ng} / \mathrm{mL}$, which is at an ultra-low level. This dose response as well as the calibration demonstrates a quantitative performance of the sensor, with a wide linear range

a

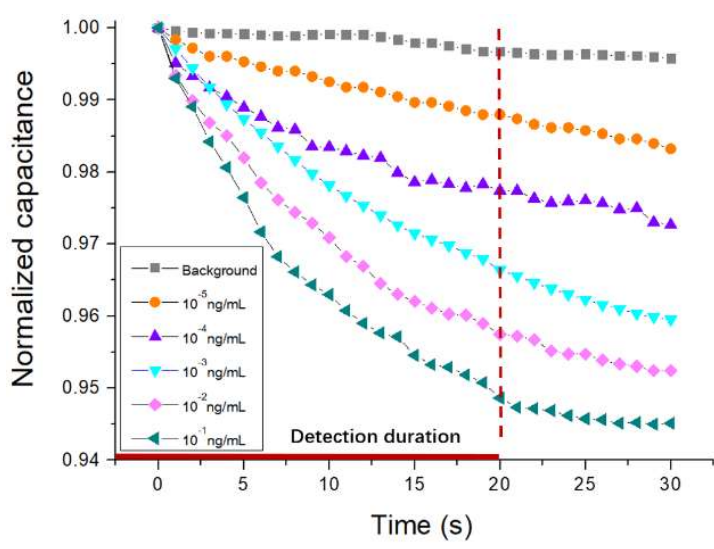

b

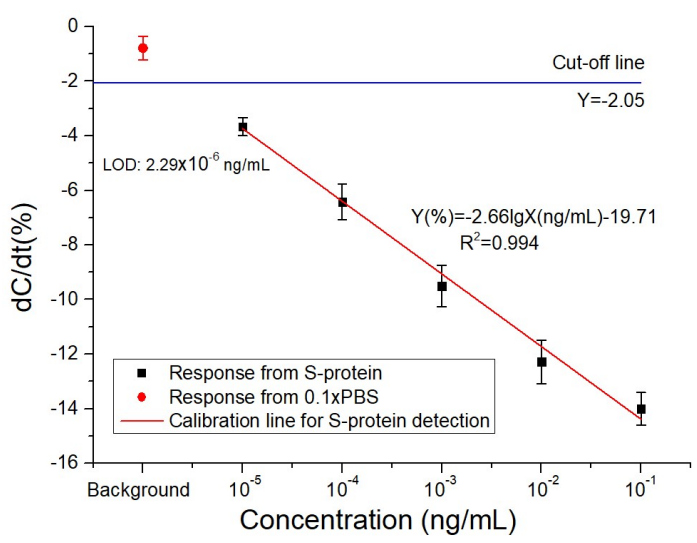

Fig. 1| Response acquired from the immunosenors. a, The typical transient capacitance normalized by its initial value changing with time. Five concentrations of S-protein (from $10^{-5}$ to $10^{-1} \mathrm{ng} / \mathrm{mL}$ ) as well as the background of $0.1 \times$ PBS are continuously detected for $30 \mathrm{~s}$. The appearance of worse linearity helps to decide the final detection duration. The equivalent slope of every curve is defined as $\mathrm{dC} / \mathrm{dt}$. $\mathbf{b}$, Dose response and its calibration. The triplicate data are acquired from three freshly prepared sensors, and the error bar is their standard deviation. Plugging the cut-off value into the calibration equation, the LOD can be calculated. 
Selectivity of S-protein against the interferences. For a qualified sensor, selectivity for the target is the most important performance. In this work, the acquired effective response is verified in three ways: (1) comparing the response from functionalized sensors with that from dummy sensors when S-protein is test, (2) comparing the response from S-protein with that from the background of $0.1 \times \mathrm{PBS}$, and (3) comparing the response from S-protein with that from the interferences. Here the dummy sensor is defined for an IDME chip without antibody functionalization to verify the probe's effectiveness.

The comparison result is given in Fig. 2a, where the response from background is within 1\%, the dummy sensors keep unresponsive, and the three interference molecules of SARS-CoV-2 nucleocapsid (N-) protein, peptidoglycan (PGN), and lipopolysaccharide (LPS) all cause unobvious response. In Fig. 2a, the biggest response is from the N-protein of SARS-CoV-2 especially when its concentration is high. The maximum response of $-4.04 \%$ from N-protein at $10^{-1} \mathrm{ng} / \mathrm{mL}$ is equivalent to the response from S-protein at $4.27 \times 10^{-4} \mathrm{ng} / \mathrm{mL}$ calculated using the calibration line, then the selectivity ratio of this sensor is calculated to be $234: 1\left(10^{-1} \mathrm{ng} / \mathrm{mL}: 4.27 \times 10^{-4} \mathrm{ng} / \mathrm{mL}\right)$. Because Nprotein is also a valuable marker for SARS-CoV-2 recognition, a weak response can help recognize the target in deed. Then, the real selectivity ratio should be obtained with PGN response, which is most significant $(-1.68 \%)$ except $\mathrm{N}$-protein also at $10^{-1} \mathrm{ng} / \mathrm{mL}$. Using the similar method, the selectivity ratio is calculated to be about $6.02 \times 10^{5}: 1$.

To demonstrate the capability of target recognition in hybrid matrices, S-protein detection is also performed in $0.1 \times$ PBS containing peptidoglycan (PGN) and lipopolysaccharide (LPS) both of $10^{-3} \mathrm{mg} / \mathrm{mL}$, which most possibly exist at the food surface dissociated from various bacteria. In this test, the spiked S-protein is of $10^{-4} \mathrm{ng} / \mathrm{mL}$. As shown in Fig. 2b, although the concentrations of nontarget molecules are 10 fold higher than that of S-protein, the response is only a bit larger than that in PBS without impurities, and still within an acceptable tolerance from the calibration curve. The response is about $107.94 \%$ of the calibrated concentration, meeting the requirement of practical quantitative detection in hybrid matrices. 

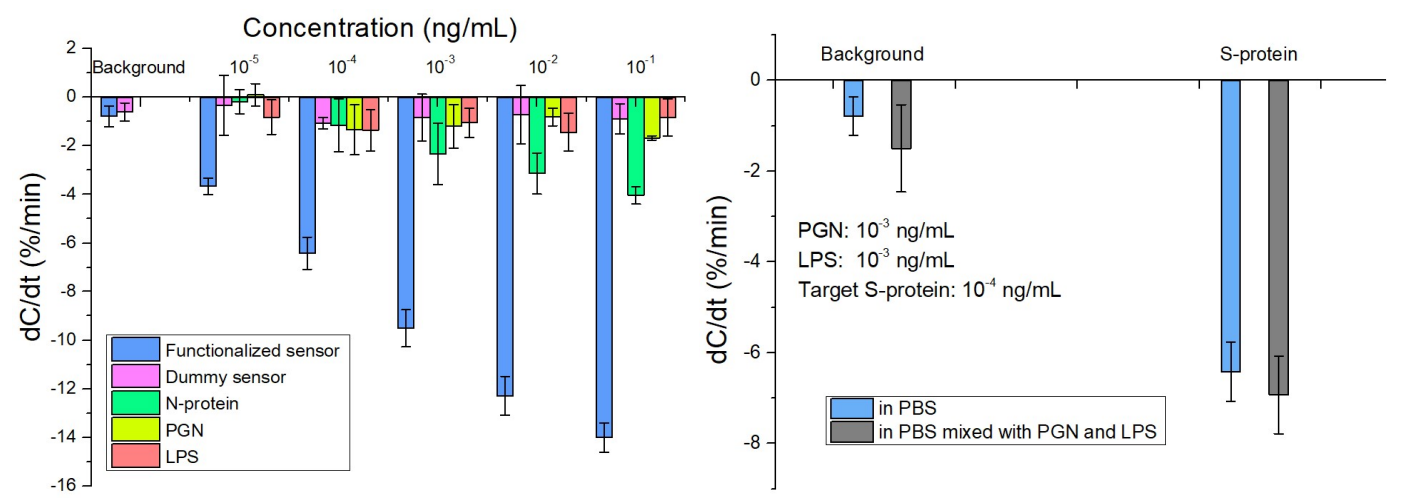

Fig. 2| Specificity verification of the immunosenors. a, Specificity verification using different types of sensors and different analytes. The background is first tested to verify the blank control and the sensor blocking effect. Functionalized sensors are compared with dummy sensors (also blocked with lactalbumin), to characterize the antibody functionalization. The control group contains nucleocapsid (N-) protein, peptidoglycan (PGN) and lipopolysaccharide (LPS). b, Specificity verification in hybrid medium. A hybrid medium is constructed by mixing PGN and LPS in $0.1 \times P B S$ solution. The PGN and LPS concentrations in $0.1 \times$ PBS are both $10^{-3} \mathrm{ng} / \mathrm{mL}$, and the spiked S-protein is at $10^{-4} \mathrm{ng} / \mathrm{mL}$.

Detection of S-protein in practical media. After verification of the sensor performance as well as the test strategy, we have applied this approach in practical media for trace S-protein detection. Three types of media associated with cold-chain food are modeled: melted tap water from the ice for cold-chain transportation, extracts from different fresh seafood surfaces, and soaking liquid from a packing bag for frozen food. Because the conformed food samples contaminated by SARS-CoV2 are extremely difficult to obtain, different matrices spiked with S-protein are detected.

The S-protein detection in the tap water is shown in Fig. 3a, where the spiked concentration is from $10^{-5}$ to $10^{-1} \mathrm{ng} / \mathrm{mL}$. For comparison, the detection result from spiked $0.1 \times \mathrm{PBS}$ is provided together. In Fig. 3a, the response from the tap water is lower than from PBS. According to this figure, the background of the tap water is above zero, which may be caused by very few large particles such as bacteria and dust in the solution. If the adsorbed particles are too few in number and the particle size is large (up to $\mathrm{nm}$ or $\mu \mathrm{m}$ ), the interfacial capacitance will increase as illustrated in supplementary information (Fig. S2). In Fig. 3a, although the baseline of the tap water is above zero, 
the variation tendency of response keeps a good agreement with that from spiked PBS, showing an expected dose relationship. For practical application, the response will reflect the real S-protein concentration after a simple baseline calibration.

a

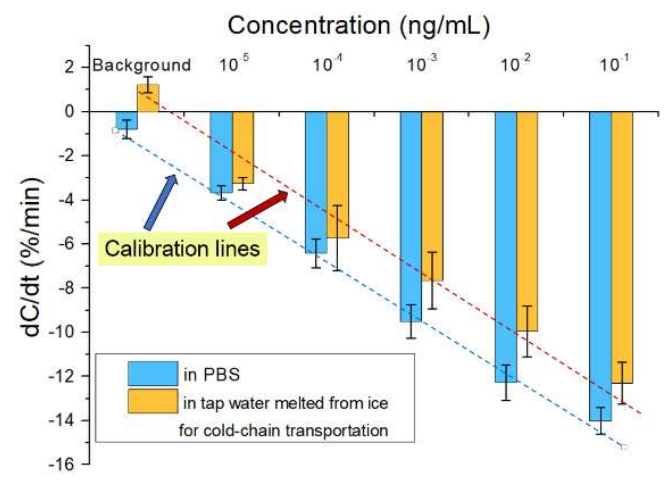

b

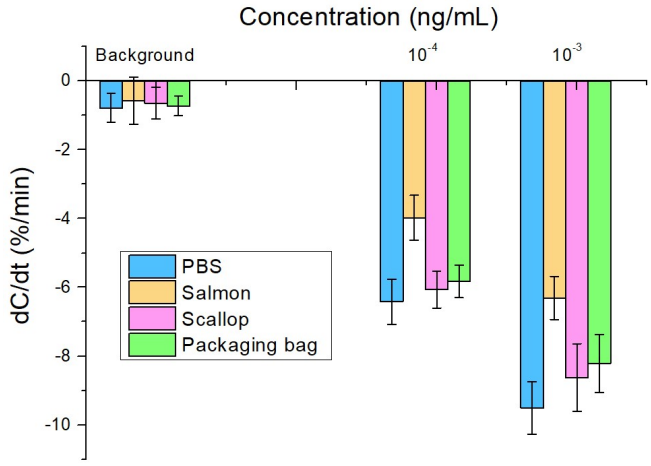

Fig. 3| S-protein detection in practical samples. a, Dose response from S-protein in melted tap water from the ice for cold-chain transportation. 5 concentrations from $10^{-5}$ to $10^{-1} \mathrm{ng} / \mathrm{mL}$ are spiked and detected in the tap water, and the calibration is performed as a red dash line. The blue dash line as a standard calibration in PBS is provided for comparison. b, S-protein detection in 3 samples associated with cold-chain food. Leaching solutions are obtained from salmon, scallop and packing bag for beef, all having two theoretical concentrations $\left(10^{-4}\right.$ and $\left.10^{-3} \mathrm{ng} / \mathrm{mL}\right)$. The corresponding backgrounds are as a quality control for the sensors before application. The response in $0.1 \times \mathrm{PBS}$ is provided as a reference.

Other detections for S-protein associated with cold-chain food are shown in Fig. 3b, with three types of materials, i.e., salmon, scallop and a packing bag for frozen beef. Here two concentrations of $10^{-4}$ and $10^{-3} \mathrm{ng} / \mathrm{mL}$ are obtained by spiking, incubation and dilution steps as introduced in the section of methods. To get rid of the impurity such as lipid particles, centrifugation is also executed before final detection. In Fig. 3b, four backgrounds all show negligible response around 1\% of dC/dt, indicating a qualified sensor preparation to block nonspecific adsorption. The target S-protein in various matrices can be distinctly recognized at both two concentrations, although the responses are smaller than that from PBS solution. In these results, the smallest response is from the salmon. Because the salmon sample has been processed in strips, and the lipid is rich at the surface, there 
are many organic molecules and particles dispersed and suspended in the turbid solution. Although high-speed centrifugation has made the background unresponsive, the positive response may be inhibited in some degree. For further practical application with these complex samples, a new calibration can be made for accurate quantitative determination of SARS-CoV-2 contaminant. By contrast, surface of the scallop and packing bag is much more smooth and dense, so that the interference is much less, and the S-protein can be more completely dispersed in PBS solution. As a result, the response from these two samples are more notable.

According to these results, S-protein in different cold-chain food samples can be successfully detected by our sensor method.

\section{Conclusions}

As a key target for SARS-CoV-2 recognition, S-protein is showing great value for the virus recognition besides drug and vaccine development. In this work, a strategy for real-time, selective, and low-cost detection of S-protein from SARS-CoV-2 is presented based on a low-cost commercial IDME based sensor combined with interfacial capacitance sensing method. An anti-SARS-CoV-2 S-protein antibody is employed as a bio-probe immobilized on the IDME surface to specifically recognize the trace S-protein in cold-chain food associated samples.

Due to effective utilization of DEP force, S-protein particles are attracted towards the sensor's surface and captured by the antibodies in $20 \mathrm{~s}$. Also, the response acquisition is completely integrated into the target enrichment process, thus the response time is shorten to $20 \mathrm{~s}$, a duration meeting the real-time detection demand on site. Besides, the LOD of this detection reaches an ultralow level, i.e., $10^{-6} \mathrm{ng} / \mathrm{mL}$. Meanwhile, the linear range of $\left(10^{-5} \sim 10^{-1} \mathrm{ng} / \mathrm{mL}\right)$ is extremely wide covering the possible range of S-protein concentration at the food surface. Even when testing a food sample with complex surface such as salmon strip, the presence and concentration of S-protein can be easily determined. In fact, cold-chain foods are mostly contaminated with SARS-CoV-2 virions by the workers carrying virus in food-processing factories. Because virus replication cannot occur at the food surface, the level of virus concentration should be very low, which poses a great challenge to traditional detection method. As such, the ultra-low LOD of the developed strategy shows an immense advantage in trace virus marker detection.

Another merit of this strategy is the high specificity. The selectivity for S-protein against the 
interference is calculated to be $6.02 \times 10^{5}: 1$, which means that the response from non-target molecules is equal to that from the target when their concentration ratio reaches $10^{5}$. Not only common organic molecules, but also various matrices as backgrounds are verified to be nonresponsive, with known or unknown components. Therefore, the sensor has demonstrated the required specificity to identify the S-proteins from practical samples with complex backgrounds.

The cost for a single sensor device is estimated to be about 1 dollar. As a result, this sensor is designed to be a disposable chip. Meanwhile, the impedance analyzer for capacitance detection is also inexpensive and lightweight. As discussed previously, the detection operation as well as the pretreatment is simple only including dilution and centrifugation steps. Therefore this sensor can be operated by a lay person. Based on the above characteristics, the platform and method for Sprotein detection provides a promising solution for large-scale applications in cold-chain food quarantine requiring quick response, low limit of detection, high specificity, user-friendly operation and low cost as well.

\section{Methods}

Target enrichment and sensing mechanism. For trace particle detection in liquid, the pre-concentration of target particles is always crucial to a successful test [33-35]. Compared with most techniques for pre-concentration needing an extra incubation or equipment, $\mathrm{AC}$ electrokinetic (ACEK) effects can manipulate nanoparticles efficiently to realize target enrichment rapidly without extra processes or devices [36-38]. For relatively large molecules such as proteins, dielectrophoresis (DEP) force, an important ACEK effect, has been demonstrated as a dominant force applied on the particles $[39,40]$. In this work, an IDME chip is used as a physical device to induce DEP effect. DEP force can be expressed as shown as Eq. S1 in the supplementary information, affected by the electric field gradients in the liquid. According to Eq. S1, the voltage applied to the IDME has a positive correlation to the DEP force.

When an electrode is immersed in a solution, electric double layer (EDL) will appear due to the accumulated charges at the electrode surface and the layer of induced counter ions above the surface [41,42]. As shown in Fig. S3, the layer of antibody and lactoalbumin molecules at the IDME surface plus the EDL forms the initial dielectric layer of the interfacial capacitance. When S-proteins are captured by the antibodies, the dielectric layer becomes thicker, and as a result, the interfacial capacitance becomes smaller as deduced in Eq. S2. The change of normalized capacitance per minute, i.e., $\mathrm{dC} / \mathrm{dt}(\% / \mathrm{min})$, directly reflects the S-protein adsorption level, by which the S-protein 
concentration in solution can be indicated. Using an IDME of micron scale, $\mathrm{dC} / \mathrm{dt}$ is a competitive parameter to reflect tiny change at the electrode interface $[30,37,43]$. Therefore, ultra-trace S-protein detection can be expected. In practice, the lab-prepared sensors may have inconsistency due to different effective electrode surface and total recognition sites. Here, the normalization by initial capacitance, as described in Eq. S2, has no relation with the initial electrode surface, thus can minimize the deviation between the response from different sensors.

Materials and reagents. The S-protein (S1 subunit) is purchased from Cellregen (Beijing) Life Science and Technology Co., LTD, which is recombinant and expressed by prokaryotic system with the host of Escherichia coli. The molecular weight this protein is $75.3 \mathrm{kDa}$, with a purity above $90 \%$. The sodium dodecyl sulfate polyacrylamide gel electrophoresis (SDS-PAGE) result is provided in Fig. S4a, and the amino acid sequence is shown in Fig. S4b. The anti-SARS-CoV-2 spike protein S1 (mouse monoclonal $\mathrm{IgG}$ ) is purchased from Anygo Technology Co., LTD, China, with a purity above $95 \%$. The recombinant N-protein is also provided by Cellregen (Beijing) Life Science and Technology Co., LTD, and the LPS (L2880) is ordered from Sigma-Aldrich Co., LTD. The PGN is bought from Nanjing Duly Biotechnology Co. LTD, China. The IDME chips are modified based on commercially available surface acoustic wave (SAW) chips (AVX Corps' KYOCERA 433K).

Sensor preparation and test protocol. The SAW chip is packaged by a ceramics chamber with the outer size of $5 \times 3.5 \mathrm{~mm}$, as shown in Fig. S5a. The chamber is enclosed by a metal cover. Inside the chamber, an aluminum IDME structure is fabricated on a ceramic substrate. As shown in Fig. S5b, the widths of finger and gap of the IDME are 2 $\mu \mathrm{m}$ and $1.5 \mu \mathrm{m}$, respectively. For sensor preparation, the metal cover is first removed, and the chip is thoroughly cleaned by soaking in acetone for $25 \mathrm{~min}$, soaking in isopropyl alcohol for $2 \mathrm{~min}$ and rinsing with purified water for $10 \mathrm{~s}$. Then, the chip is treated with ozone for $30 \mathrm{~min}$ to improve the hydrophilia of the electrode surface. After that, $10 \mu \mathrm{L}$ antibody ( $10 \mu \mathrm{g} / \mathrm{mL}$ in $0.05 \times \mathrm{PBS})$ is added into the chamber and incubated for $24 \mathrm{~h}$ in a humidor. The final step for is blocking for $3.5 \mathrm{~h}$ using lactoalbumin $(100 \mu \mathrm{g} / \mathrm{mL}$ in $0.05 \times \mathrm{PBS})$ to cover the unfunctionalized IDME area.

To verify the functionalization on the IDME, X-ray photoelectron spectroscopy (XPS) and electrical impedance spectra are employed to characterize the change at the IDME surface, as described in Fig. S6a, Fig. S6b and Fig. S6c. The XPS energy spectra in Fig. S6a have confirmed that antibody immobilization step has caused the element change at the IDME surfacecan be. Furthermore, the antibody and lactalbumin as an added dielectric layer is clearly shown through the Bode plot, where Fig. S6b demonstrates an impedance increase due to the dielectric layer, and Fig. S6c shows the change of interfacial capacitance though phase angle. The blocking changes little of the electrical 
parameters, which is due to the little change of the dielectric layer thickness as shown in Fig. S3. These characterization means are taken as a quality control method for the sensor's functionalization.

The functionalized sensor is first connected to an impedance analyzer (TH2829C, Tonghui Electronic Co. LTD). After the sample is dropped into the sensor's chamber, an AC signal is applied for a selected duration of $20 \mathrm{~s}$. The measurement system with this sensor is shown in Fig. S7. The frequency of the AC signal is set to be $100 \mathrm{kHz}$ according to previous study $[28,41]$, and the voltage is optimized to be $100 \mathrm{mV}$ according to the optimization in Fig. S8. Based on the measured capacitance, $\mathrm{dC} / \mathrm{dt}$ can be calculated and analyzed. To demonstrate the sensor's repeatability and consistency, all the data are presented with error bars, which represent the standard deviation obtained from three sensors of the same batch.

Before measurement, all the practical samples should be pretreated. The conductivity of raw tap water (melted from the ice for cold-chain transportation) is tested to be $0.012 \mathrm{~S} / \mathrm{m}$. Then the raw tap water is 1 : 1 mixed with $0.19 \times \mathrm{PBS}$ to obtain a mixture with the conductivity of $0.141 \mathrm{~S} / \mathrm{m}$, which is as same as that of $0.1 \times \mathrm{PBS}$. During all the detection in this work, the solution conductivity is kept to be $0.141 \mathrm{~S} / \mathrm{m}$, which makes an equal effect on DEP by solution conductivity $[28,29]$. Then S-protein is added in this mixed background also called as "tap water". For cold-chain-food associated samples (salmon, scallop and a packing bag for frozen beef), a small piece of certain sample about $8 \times 8 \mathrm{~mm}$ is first cut off, and $10 \mu \mathrm{L}$ S-protein of $1 \mathrm{ng} / \mathrm{mL}$ is spread over it. After the solid sample is incubated for $12 \mathrm{~h}$ in a closed tube at $4{ }^{\circ} \mathrm{C}, 990 \mu \mathrm{L} 0.1 \times \mathrm{PBS}$ is added into the tube. Then the solution is sufficiently agitated and centrifuged at $3000 \mathrm{rpm}$ for $10 \mathrm{~min}$. The supernatant from this mixture with a theoretical S-protein concentration of $10^{-2} \mathrm{ng} / \mathrm{mL}$ is ready for further dilution to obtain the final samples with theoretical concentrations of $10^{-3}$ and $10^{-4} \mathrm{ng} / \mathrm{mL}$. During the pretreatment for salmon slice, an additional centrifugation at $5000 \mathrm{rpm}$ for 10 min is necessary to remove the abundant interference, and the floating lipid layer should be excluded when the supernatant is collected. To obtain the corresponding backgrounds for these practical samples, similar process is performed except for the S-protein spreading and incubation steps.

\section{References}

[1] Bachofen, C. Selected viruses detected on and in our food. Curr. Clin. Micro. Rpt. 5, 143-153 (2018).

[2] Severi, E., Verhoef, L., Thornton, L., Guzman-Herrador, B. R. \& Rizzo, C. Large and prolonged food-borne multistate hepatitis A outbreak in Europe associated with consumption 
of frozen berries, 2013 to 2014. Euro Surveill. 20, 21192 (2015).

[3] Yoon, Y. K., et al. Epidemiological and genetic analysis of a sustained communitywide outbreak of hepatitis A in the Republic of Korea, 2008: a hospital-based case-control study. $J$. Clin. Virol. 46, 184-188 (2009).

[4] Hall, A. J. Noroviruses: The perfect human pathogens? J. Infect. Dis. 205, 1622-1624 (2012).

[5] Somura, Y., et al. Detection of norovirus in food samples collected during suspected foodhandler-involved foodborne outbreaks in Tokyo. Lett. Appl. Microbiol. 69, 175-180 (2019).

[6] Alhadrami, H. A., et al. Development of a simple, fast, and cost-effective nanobased immunoassay method for detecting norovirus in food samples. ACS Omega 5, 12162-12165 (2020).

[7] Bosch, A. et al., Analytical Methods for Virus Detection in Water and Food. Food Anal. Methods 4, 4-12 (2011).

[8] Rajapaksha, P., et al. A review of methods for the detection of pathogenic microorganisms. Analyst 144, 396-411 (2019).

[9] Velavan, T. P. \& Meyer, C. G. The COVID epidemic. Trop. Med. Int. Health. 25, 278-280 (2020).

[10] Ceylan, Z., Meral, R. \& Cetinkaya, T. Relevance of SARS-CoV-2 in food safety and food hygiene: potential preventive measures, suggestions and nanotechnological approaches. Indian J. Virol. 31, 155-160 (2020).

[11] Roos,Y. H. Water and Pathogenic Viruses Inactivation - Food Engineering Perspectives. Food Eng. Rev. 12, 251-267 (2020).

[12] Aiyar, A. \& Pingali, P. Pandemics and food systems - towards a proactive food safety approach to disease prevention \& management. Food Secur. 3, 749-756 (2020).

[13] Doremalen, N. V., Bushmaker, T., Morris, D. H., Holbrook, M. G. \& Munster, V. J. Aerosol and surface stability of SARS-CoV-2 as compared with SARS-CoV-1. New Engl. J. Med. 382, $1564-1567$ (2020).

[14] Exploring wet markets. Nat. Food 1, 241 (2020). https://doi.org/10.1038/s43016-020-0090-1

[15] Rajiuddin, S. M., Jensen, T., Hansen, T. B. \& Schultz, A. C. An optimised direct lysis method for viral RNA extraction and detection of foodborne viruses on fruits and vegetables. Food Environ. Virol. 12, 226-239 (2020). 
[16] Chen, J., Wu, X., Sanchez, G. \& Randazzo, W. Viability RT-qPCR to detect potentially infectious enteric viruses on heat-processed berries. Food Control 107, 106818 (2019).

[17] Hennechart-Collette, C., Niveau, F., Martin-Latil, S., Fraisse, A. \& Perelle, S. Development of an extraction method to detect enteric viruses in dressed vegetables. Int. J. Food Microbiol. 311, 108349 (2019).

[18] Boxman, I. L. A., et al. Detection and quantification of hepatitis E virus RNA in ready to eat raw pork sausages in the Netherlands. Int. J. Food Microbiol. 333, 108791 (2020).

[19] Zhuang, J., Yin, J., Lv, S., Wang, B. \& Mu, Y. Advanced "lab-on-a-chip" to detect viruses Current challenges and future perspectives. Biosens. Bioelectron. 163, 112291 (2020).

[20] Cheng, C., Cui, H., Wu, J., \& Eda, S. A PCR-free point-of-care capacitive immunoassay for influenza a virus. Microchim. Acta 184, 1649-1657 (2017).

[21] Cheng, C., et al. Unamplified RNA sensor for on-site screening of Zika virus disease in a limited resource setting. ChemElectroChem 4, 485-489 (2017).

[22] Wrapp, D., et al. Cryo-EM structure of the 2019-nCoV Spike in the prefusion conformation. Science 367, 1260-1263 (2020).

[23] Walls, A. C., et al. Structure, function, and antigenicity of the SARS-CoV-2 spike glycoprotein. Cell 180, 281-292 (2020).

[24] Ou X., et al. Characterization of spike glycoprotein of SARS-CoV-2 on virus entry and its immune cross-reactivity with SARS-CoV. Nat. Commun. 11, 1620 (2020).

[25] Zhang X., et al. Electrical probing of COVID-19 spike protein receptor binding domain via a graphene field-effect transistor. (2020). arXiv: 2003.12529v1

[26] Mavrikou, S., Moschopoulou, G., Tsekouras, V. \& Kintzios, S. Development of a portable, ultra-rapid and ultra-sensitive cell-based biosensor for the direct detection of the SARS-CoV2 S1 Spike Protein Antigen. Sensors 20, 3121 (2020).

[27] Ahmadivand, A. et al. Femtomolar-level detection of SARS-CoV-2 spike protein using toroidal plasmonic metasensors. 2020. arXiv: 2006.08536

[28] Chen, R., Huang, X., Xu, H., Xiong, Y., \& Li, Y. Plasmonic enzyme-linked immunosorbent assay using nanospherical brushes as a catalase container for colorimetric detection of ultralow concentrations of Listeria monocytogenes. ACS Appl Mater Inter. 7, 28632-28639 (2015).

[29] Jayan, H., Pu, H., Sun, D. W. Recent development in rapid detection techniques for 
microorganism activities in food matrices using bio-recognition: A review. Trends Food Sci. Tech. 95, 233-246 (2020).

[30] Zhang, J., et al. An on-site, highly specific immunosensor for Escherichia coli detection in field milk samples from mastitis-affected dairy cattle. Biosens. Bioelectron. 165, 112366 (2020).

[31] Qi, H., et al. Rapid detection of trace $\mathrm{Cu}^{2+}$ using an L-cysteine based interdigitated electrode sensor integrated with AC electrokinetic enrichment. Electrophoresis 40, 2699-2705 (2019),

[32] Zhang, J., et al. An interdigitated microelectrode based aptasensor for real-time and ultratrace detection of four organophosphorus pesticides. Biosens. Bioelectron. 150, 111879 (2020).

[33] Myint, M. S., Johnson, Y. J., Tablante, N. L. \& Heckert, R. A. The effect of pre-enrichment protocol on the sensitivity and specificity of PCR for detection of naturally contaminated salmonella in raw poultry compared to conventional culture. Food Microbiol. 23, 599-604 (2006).

[34] Nguyen, N. V. \& Jen, C. P. Impedance detection integrated with dielectrophoresis enrichment platform for lung circulating tumor cells in a microfluidic channel. Biosens. Bioelectron. 121, $10-18(2018)$.

[35] Wang, Q., et al. Enhancing host-cell protein detection in protein therapeutics using HILIC enrichment and proteomic analysis. Anal. Chem. 92, 10327-10335 (2020).

[36] Cui, H., et al. Rapid and sensitive detection of small biomolecule by capacitive sensing and low field AC electrothermal effect. Sensor. Actuat. B-Chem. 226, 245-253 (2016).

[37] Zhang, J., et al. Rapid detection of ultra-trace nanopaticles based on ACEK enrichment for semiconductor manufacturing quality control. Microfluid. Nanofluid. 23, 2 (2019).

[38] Ivanoff, C. S., et al. AC electrokinetic drug delivery in dentistry using an interdigitated electrode assembly powered by inductive coupling. Biomed. Microdevices 18, 84 (2016).

[39] Liu, X., Cheng, C., Jayne, W., Eda, S. \& Guo, Y. A low cost and palm-size analyzer for rapid and sensitive protein detection by AC electrokinetics capacitive sensing. Biosens. Bioelectron. 90, $83-90$ (2016).

[40] Cheng, C., Wu, J. \& Chen, J. A highly sensitive aptasensor for on-site detection of lipopolysaccharides in food. Electrophoresis 40, 890-896 (2018).

[41] Morgan, H. \& Green N. G. AC electrokinetics: colloids and nanoparticles. Research Studies 
Press (2002).

[42] Oueslati, R., Cheng, C., Wu, J. \& Chen, J. Highly sensitive and specific on-site detection of serum cocaine by a low cost aptasensor. Biosens Bioelectron. 108, 103-108 (2018).

[43] Zhang, J., et al. Rapid, highly sensitive detection of Gram-negative bacteria with lipopolysaccharide based disposable aptasensor. Biosens. Bioelectron. 112, 48-53 (2018).

\section{Acknowledgments}

This work was supported by the National Key R\&D Program of China (2016YFD0401104, 2017YFC1600603, 2019YFC1605302), the National Natural Science Foundation of China (62074047), Anhui Provincial Natural Science Foundation of China (1908085MF180), and the open research fund of the Key Laboratory of MEMS of the Ministry of Education, Southeast University, China. J. Wu thanks the support from the University of Tennessee Initiative of PON/POC Nanobiosensing, Institute of Secure and Sustainable Environment and USDA NIFA (Grant No. 2017-67007-26150).

\section{Author contributions}

J.Z., J.W. and L.Z. designed the research. H.Q., J.Z., X.F., W.Z., F.Y. and Y.C. performed the research. H.Q. and J.Z. analysed the data. H.Q., J.Z. and J.W. wrote the paper.

\section{Competing interests}

The authors declare no competing interests. 